\title{
Contour Completion without Region Segmentation
}

\author{
Yansheng Ming, Hongdong Li, Member, IEEE, and Xuming He, Member, IEEE
}

\begin{abstract}
Contour completion plays an important role in visual perception, where the goal is to group fragmented low-level edge elements into perceptually coherent and salient contours. Most existing methods for contour completion have focused on pixelwise detection accuracy. In contrast, fewer methods have addressed the global contour closure effect, despite of psychological evidences for its importance. This paper proposes a purely contour-based higher-order CRF model to achieve contour closure, through local connectedness approximation. This leads to a simplified problem structure, where our higher-order inference problem can be transformed into an integer linear program (ILP) and be solved efficiently. Compared with methods based on the same bottom-up edge detector, our method achieves a superior contour grouping ability (measured by Rand index), a comparable precision-recall performance, and more visually pleasing results. Our results suggest that contour closure can be effectively achieved in contour domain, in contrast to a popular view that segmentation is essential for this purpose.
\end{abstract}

Index Terms-contour detection, closure principle, conditional random field.

\section{INTRODUCTION}

I $\mathrm{N}$ the Oxford dictionary, a contour is defined as an outline representing or bounding the shape or form of something. As a fundamental vision problem, the contour detection problem seeks to extract curves representing object shapes from images. Solving this problem can help visual systems to group pixels into objects and reveal their semantic information [45].

Although several studies on contour detection have focused on point-wise detection accuracy, our paper and several others emphasize the topological properties of contours, contour closure in particular. The closure principle has been observed by numerous psychological studies. In [45], this principle is summarized as "all else being equal, the elements which form closed figure tend to be grouped together". Using carefully designed stimuli, Kovacs and Julesz [26] demonstrated that a set of contour fragments were more easily perceived from a noisy background if they were closed. Therefore, " a closed curve is much more than an incomplete one”.

In addition to its psychological relevance, enforcement of the closure principle could produce more meaningful contours. Given the noise and ambiguities, even state-of-the-art local edge detectors can assign low probabilities to some of the true contour segments. Consequently, contours often break into fragments after thresholding. The bottom left image of Figure 1 shows a thresholded edge map of the $\mathrm{Pb}$ detector. This image is in sharp contrast to the human labeling map at the top right in terms of contour closure. This problem undermines the ability to recall all edge points and causes difficulties in object and scene understanding. In structuralism, contours belonging

- Y. Ming is with the college of engineering and computer science, Australian National University, Canberra, 0200. E-mail: yansheng.ming@anu.edu.au

- H. Li is with with Australian National University, Canberra, 0200. E-mail: hongdong.li@anu.edu.au

- X. He is with NICTA, Canberra,0200. E-mail: xuming.he@nicta.com.au

- The first version of this work appeared in CVPR 2012 [42]. to one object can be decomposed into several interconnected parts. When these structures are smeared in contour maps, object representations can be difficult to learn in a bottom-up fashion.

Unlike other relatively local Gestalt principles, the closure principle is "more global"; thus, the enforcement of such a condition is not a simple task. Notably, most contour-grouping methods still tend to produce isolated or disconnected curve segments [55], [25]. Although some methods aim to enforce this principle [66], [37], these methods often do so at the individual contour level, thereby making them unsuitable for general natural images teeming with occlusion.

Recognizing this difficulty, Palmer [45] suggested that contours be completed by performing image segmentation, e.g. by [60]. Naturally, the boundaries of segmented regions are closed. A recent work [1] also enforced contour closure through an exponential number of constraints based on superpixel segmentation.

Despite the merits of region-based processing, the alternative contour-based approach is interesting to explore. First, several psychological phenomena, such as illusionary contours, suggest that such a mechanism exists in the human vision system. That is, contour plays a critical role in the perception of a region or shape, although the intensities within each region do not provide informative cues. The contour-based grouping is also complementary and beneficial to region-based ones. Specifically, contour-based segmentation has been widely used to understand line drawing and to process medical images, where the region-based image cues can be noisy or non-informative.

To this end, this paper presents a purely contour-based method that can produce highly completed contours. We believe our results improve the current understanding of this perceptual grouping process. Our method addresses the closure principle by proposing two contour completion hypotheses that bridge the gaps between locally detected edges. A set 


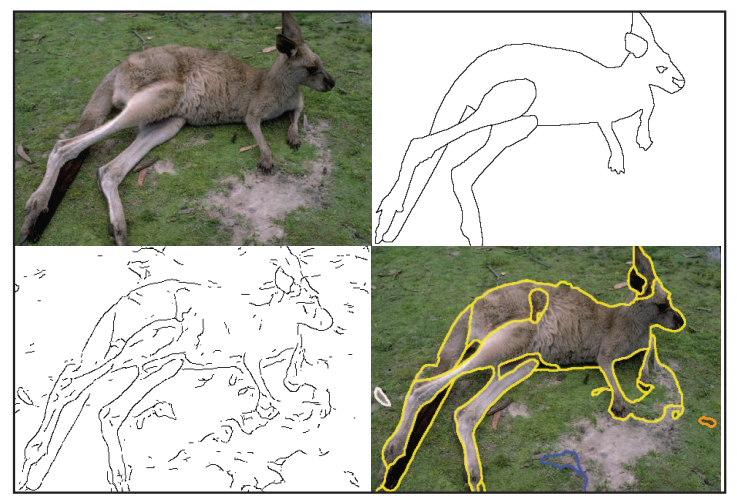

Fig. 1. The goal of this paper is to extract perceptually-salient and closed contours from images. Top Left: An image of a kangroo in the BSDS dataset. Top Right: A human-labeled contour image. Bottom Left: The contour map by $\mathrm{Pb}$, as the input to our method [41]. Bottom Right: The contour map by our method.

of contour connectedness conditions are used to ensure that each edgelet is connected to some of its neighbors. Although these connectedness conditions are only necessary and not sufficient conditions for the closure principle, this approximation generates a more efficient model, which often produces closed contours in practice. In addition to the closure principle, the principles of good continuity and proximity are also modeled by our energy function.

Collectively, our model is presented as a higher-order CRF model [13], [12] based on a novel representation of image contours and their interactions. Our energy function includes unitary potentials that reflect the local edge contrast, junction potentials that encode the continuity property, closure potentials that enforce correct contour topology, and a complexity potential that controls the level of details in the outputs.

The higher-order nature of our model originates from the connected conditions, which take all the edgelets in a neighborhood into account. Figure 6 shows that up to 25 edgelets may be involved in an energy term. In addition, the appendix A shows that our energy function is generally non-submodular. We make the inference efficient by using the connectedness constraints to reduce some of the higher-order potential functions into a linear order, which enables us to formulate the CRF inference as an integer linear programming (ILP) problem. The remaining high-order potentials are represented by linear inequalities. An efficient algorithm is further devised to determine a locally optimal integer solution of the ILP, thereby taking advantage of the specialty of our ILP problem. In our experiments, the solution energies are very close to the lower bounds.

We tested our method on synthetic data and real images. Experiments show that the method extracts multiple connected contours without loose ends, in accordance with our previous theoretical assessment. Our results are compared with several closely-related methods in terms of the pixel-wise accuracy to show that our method does not complete contours in a native or blind manner. In the BSDS300 and BSDS500 datasets, our method is advantageous under the standard precision- recall metric and a newly developed metric for grouping error. Finally, the extracted connected contours appear clean and visually pleasing, thereby suggesting that the results are probably closer to human perception. In summary, our results show that the closure principle can be enforced effectively in the contour domain. Further studies of other Gestalt factors, such as the influence of past experience, are necessary to determine the computational nature of contour detection.

\section{Previous work}

In Marr's theory, contour detection is completed at the stage of extracting the full primal sketch [39], [15]. However, more recent studies have shown that contour detection problem, as part of the perceptual grouping process, is not completed in one shot [46]. Various information and constraints can be used at different stages of contour detection.

Classical methods often apply filters, such as the Sobel operator, to an image and search for the local maxima of filter responses as edge points [6], [40], [22]. The phase information of filter responses was also used in [28]. Learning-based methods have also been developed [41], [49], [25], [53], [35], [9]. Compared with traditional methods, such as the Canny detector [6], learning based methods can use various image cues to achieve higher robustness. Recent experiments showed that their performance is relatively close to human perception in a local edge discrimination task [73]. Contours in natural images emerge at different scales [36]; thus, pooling multiple-scale information can also improve contour detection results [52] [2]. Recently proposed holistic convolutional network is able to pool information from the whole image [71].

Although local image statistics are very informative [21], psychological phenomena, such as illusionary contours, demonstrate that perception of contours is not purely based on local information. Several methods have been proposed to improve the accuracy based on the mid-level Gestalt principles, such as proximity, continuity, and closure. Most of the grouping algorithms use the Gestalt principles of proximity and good continuity, and favor smooth transitions with short gaps. An empirical study on the BSDS dataset [54] confirmed that the boundaries in natural scene images are usually smooth despite some sharp angles. The elastic function [58], [32], [19] has been used in to model contour smoothness. These cues have also been used in a previous CRF model [55] and in contour saliency methods, especially those that considered the facilitation effect [61], [16], [63], [34], [48].

As a global property, contour closure is more difficult to enforce. In previous work, contour closure is enforced by restricting the solution to be single connected contours [10], [37], [66], [67], [57], [23]. Multiple contours have to be extracted iteratively. Another group of methods derives closed contours from boundaries of segmentation [33], [2]. For example, the GPb-owt-ucm method [2] derived contours from watershed segmentation. In comparison, our method attempts to enforce closure in the contour domain instead of resorting to hierarchical segmentation. Similar to our method, some methods require some contours to be closed [55], [25]. However, without explicit constraints, these previous methods 
often produce contours with loose ends. In a previous paper [1], contour closedness is enforced by an exponential number of linear constraints, which are based on the boundaries of over-segmented images. Their inference problem is converted to a large-scale integer program. By contrast, our method only involves a constraint set of linear complexity with respect to the number of edges, thereby producing an efficient inference algorithm.

Segmentation information has been used to detect the most salient contours in the images. In [38], contours are extracted as soft boundaries of normalized cut segmentation. In previous studies [72], [24], salient contours are extracted as one-dimensional subgraphs, with minimal entanglement with themselves and other curves. The optimization problems of these models are reduced to determine the eigenvalues and eigenvectors of random walk matrices. These methods are related to the Gestalt principles of similarity and good continuity.

Even with the extensive use of mid-level grouping cues, machines continue to lag behind the human vision system. One possibility is that high-level top-down information helps humans in finding the boundaries of recognizable objects. The inverse object detector [18] estimates object contours from the output of object detectors. Wu et al. [70] proposed a category-specific contour model by training images containing similar objects. Their model was also extended to search for contours in three dimensions [69]. Top-down information can also be incorporated in variational methods [5], [7], [50], where the objective is to find specific shapes. For more detailed discussions of contour detection, we refer interested readers to a recent survey [47].

The topological constraints of our methods produce a highorder conditional random field. Traditional methods, such as the Ising model [44], are usually built upon pairwise potentials. Given the importance of high-order statistics, more CRF models include higher-order potential functions, such as those for stereo vision [68], scene labeling [14] [30], image denoising [56], segmentation [31]. In particular, the topological prior of connectivity has been used for the interactive segmentation problem [64]. Unlike their model, our method does not require all edgelets to form a connected graph. The inference of these methods is usually performed by customized algorithms [65], [29]. However, several general methods have been proposed to reduce the high-order cliques to low-order ones [11] [20].

\section{MOdELING MULTIPLE CONTOURS}

\subsection{Overview}

In this paper, the contour completion problem is formulated as a probabilistic inference problem. Given local detection results, our model seeks the maximum a posteriori solution of the conditional probabilistic distribution of contours. The distribution encodes our prior about contours such as smoothness and connectedness. In Section-3, we describe how to construct our new CRF model, and how to use it to represent multiple image contours. Section- 4 explains the CRF's potential function design. Section-5 is devoted to the proposed

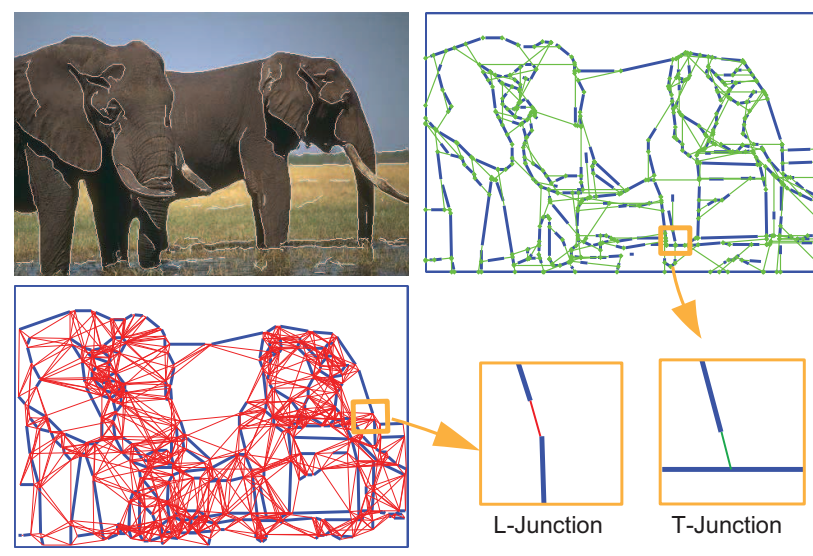

Fig. 2. Top left: An input image overlayed with its thresholded $\mathrm{Pb}$ edges. Top right: Proposed T-junction completion edgelets are shown in green, and gradient edgelets by $\mathrm{Pb}$ are shown in blue. Bottom left: Proposed L-junction completion edgelets are shown in red. Bottom right: A zoomed-in view of the completion edgelets: L-junction(left) and T-junction(right). Best viewed in color.

inference algorithm. The final two sections are experiments and conclusion.

\subsection{The boundary segment graph}

To facilitate contour completion, we need to design a proper representation that allows mid-, and long-range interactions among local edge elements. For this purpose, we first construct a graph of boundary segments based on the output of a local boundary detector, such as the $\mathrm{Pb}$ detector in [41]. On this graph, we then propose a higher-order CRF that integrates local evidences with global constraints based on the Gestalt properties of contours at a scene level.

Our graph is built in two stages. We first form a set of short boundary segments using $\mathrm{Pb}$, followed by a line fitting process. Specifically, we threshold $\mathrm{Pb}$ edge maps at the threshold of 0.05 . Then, the binary edge map was linearized with Kovesi's line fitting algorithm [27]. This algorithm will break a line at extreme points. We refer to those line segments as gradient edgelets or G-edgelets in short. Normally, there are many gaps among G-edgelets, due to occlusions and miss-detections. So at the second stage, we introduce two new types of virtual completion edgelets (or C-edgelet in short) aiming to fill in the gaps and hence complete contours.

We first shorten each gradient edgelet by several pixels at its two endpoints such that it does not connect to other gradient edgelets. The first type of completion edgelets is proposed to link two neighboring gradient edgelets with the good-continuation property, referred as L-junction edgelets. Two gradient edgelets are considered to be in the same neighborhood if the distance between two of their endpoints are smaller than a threshold (say one fifth of the image width). Ljunction edgelets connect gradient edgelets into longer contour segments by filling in the gaps caused by missing local cues and shortening. The second type of completion edgelets is used to capture the occlusion relationship between contours, referred as T-junction edgelets. A T-junction edgelet is placed 
between an endpoint of a gradient edgelet and another gradient edgelet if the extension of the former intersects the latter one without crossing other gradient edgelets. See Figure2 for an illustration of gradient and completion edgelets. Note that for each endpoint, only one T-junction edgelet is proposed, and we treat the rectangular image boundary as four gradient edgelets as they are also a kind of occluding (clipping) boundaries. Please note that there are important difference between L-junction edgelets and T-junction edgelets. An Ljunction edgelet always bridges two endpoints of gradient edgelets whereas a T-junction edgelet extends from one edgelet until it is blocked by another edgelet, as if the first boundary is occluded by the second one.

To build a graph of boundary segments, we view each edgelet as a graph node. We use the same connectivity as we propose the completion edgelets. Note that in our graph the neighboring $\mathrm{C}$-edgelets and $\mathrm{G}$-edgelets always appear alternatingly. Compared with the CDT (Constrained Delaunay Triangulation) graph proposed in [55], our graph model accepts a higher order of connectivity, and it also explicitly encodes richer types of junction relations (such as occluding/occluded) among contours. Moreover, we have experimentally confirmed that the proposed graph with about 1000 completion edges per image is able to recall $95 \%$ of the ground-truth boundary points on the BSDS300 dataset. In comparison, a CDT graph with about 300 completions per image has a recall rate of $88 \%$. Although a CDT graph seems more efficient, the high recall rate of our model is a precondition for enforcing the connectedness constraints. At the same time, the model adopt a fully factorized representation of the junction potentials, so that the model complexity only increases moderately with the number of completion edges.

\subsection{A higher-order CRF for contours}

Based on the graph of boundary segments in Section 3.2, we build a CRF model to capture both properties of individual contours, and their interactions. In particular, our model focuses on four aspects of contour properties, including 1) local image contrast; 2) contour smoothness and continuity; 3 ) contour closure; and 4) overall model-complexity.

Our model is formulated as follows. Let an image $I$ have a boundary segment graph $G=(V, E)$ with edgelets as its node set $V$. We associate a binary variable with each edgelet, and denote all variables as $\mathbf{Y}=\left\{y_{i} \in\{0,1\}, \forall i \in V\right\}$. The edgelet variables are divided into two sets: the gradient edgelets $\mathbf{Y}^{g}=\left\{y_{i}, i \in V_{g}\right\}$ and the completion edgelets $\mathbf{Y}^{c}=\left\{y_{i}, i \in V_{c}\right\}$. When we need to differentiate two types of completion edgelets, we use $V_{c}^{l}$ and $V_{c}^{t}$ to denote the sets of L-junction edgelets and T-junction edgelets, respectively. Each edgelet is connected to neighboring edgelets at the vicinity of its two endpoints. To capture the "good continuity" and "closure" rules, we need to consider both near-range and longer-range interactions between the edgelets, by introducing the following three types of cliques in the graph:

1) For every connected edgelet pair, we have a "pairwise clique", and denote the set of pairwise cliques as $C^{P}$;

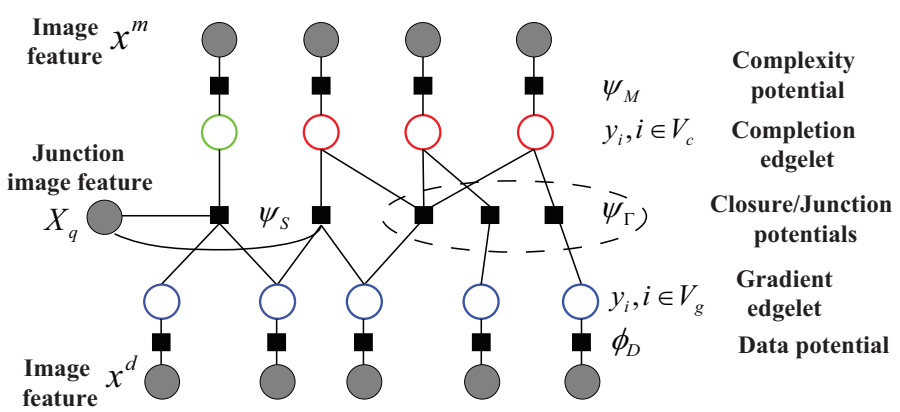

Fig. 3. A factor graph representation of our CRF model. The circles represent variables in the model and the squares are potential functions (i.e. factors). The gradient edgelets are shown in blue and the completion edgelets are in red or green. Some connections are not shown for clarity and see text for details.

2) We assign every completion edgelet (no matter it is Ltype or T-type) a triple-node clique that includes itself and two neighboring gradient edgelets, and this type of clique is called "junction clique", and the entire set of such junction-cliques is denoted as $C^{J}$;

3) At an endpoint of a given gradient edgelet, all the edgelets connecting to this endpoint induce a higherorder clique, and we refer to the set of such higher-order cliques as $C^{H}$.

Our CRF defines a joint distribution of the labels $\mathbf{Y}$ given the input observation $\mathbf{X}$, denoted as $P_{\mathbf{Y} \mid \mathbf{X}}$ for short, which includes four types of potential functions,

$$
\begin{aligned}
P_{\mathbf{Y} \mid \mathbf{X}}= & \frac{1}{Z_{\mathbf{X}}} \exp \left\{-\left(\sum_{i \in V_{g}} \phi_{D}\left(y_{i}, \mathbf{x}_{i}^{d}\right)+\sum_{q \in C^{J}} \psi_{J}\left(\mathbf{Y}_{q}, \mathbf{X}_{q}\right)\right.\right. \\
& \left.\left.+\sum_{q \in C^{P} \cup C^{H}} \psi_{\Gamma}\left(\mathbf{Y}_{q}\right)+\sum_{i \in V_{c}} \psi_{M}\left(y_{i}, \mathbf{x}_{i}^{m}\right)\right)\right\}
\end{aligned}
$$

where $Z_{\mathbf{X}}$ is the partition function, and the potential functions $\phi_{D}$ (data term), $\psi_{J}$ (junction term), $\psi_{\Gamma}$ (global closure/connectedness effect term) and $\psi_{M}$ (model-complexity term) are used to describe different aspects of desirable contour properties. We use $\mathbf{Y}_{q}$ to represent the variables associated with clique $q$.

A factor graph representation of the CRF model is shown in Figure 3. The circles represent the variables in the model and the squares are potential functions. The black circles in the uppermost layer denote local observations. In the layer below, the green circles stand for the binary random variables of gradient edgelets, while the purple circles in the bottom layer stand for the binary labels of completion edgelets. The connection between a gradient edge node and an observation node represents a unary term in our potential function, encoding the information from local edge detection.The square nodes between gradient edge nodes and completion edge nodes represent junction potential functions. The prior terms which are unary terms are not shown in this graph for clarity. 


\section{DESIGN OF POTENTIAL FUNCTIONS}

\subsection{Unary data terms $\phi_{D}$}

A unary potential $\phi_{D}\left(y_{i}, \mathbf{x}_{i}^{d}\right)$ computes the likelihood score that the $i$-th edgelet lies on a true contour. This term is defined as a linear function of a boundary feature vector $\mathbf{x}_{i}^{d}$ :

$$
\phi_{D}\left(y_{i}, \mathbf{x}_{i}^{d}\right)=\alpha \mathbf{w}_{d}^{T} \mathbf{x}_{i}^{d} y_{i},
$$

where $\mathbf{w}_{d}$ is the weight for image features and $\alpha$ is an overall weight for the data term. In this work, we use the lengths of edgelet $l_{i}$, logarithm of the average $\mathrm{Pb}$ value, and their product as input features.

\subsection{Junction potentials $\psi_{J}$}

For every completion edgelet $y_{i}, i \in V_{c}$ and the associated triple-node clique $q \in C^{J}$, we define a junction potential $\psi_{J}\left(\mathbf{Y}_{q}, \mathbf{X}_{q}\right)$ to encode the continuity property. For an Ljunction, we design an L-potential to impose the principle of good-continuity; For a T-junction, we design a T-potential to express the likelihood of occluding/occluded relationships. For either case, we define an image-dependent triplet potential function involving the central completion edgelet $y_{i}$ and its two connected neighbors $\left\{y_{j}, y_{k}\right\}$, as shown below (and in Fig-4):

$$
\psi_{J}\left(\mathbf{Y}_{q}, \mathbf{X}_{q}\right)=\psi_{J}\left(y_{i}, y_{j}, y_{k}, \mathbf{X}_{q}\right)=\mathbf{w}_{J}^{T} \mathbf{X}_{q} y_{i} y_{j} y_{k},
$$

where $\mathbf{w}_{J}$ is the coefficient for the junction feature vector $\mathbf{X}_{q}$, which is extracted from the neighborhood of clique $q$. Note that this potential assigns a score of $\mathbf{w}_{J}^{T} \mathbf{X}_{q}$ to the case that the whole triplet is active, and zero otherwise.

The image feature vectors used in this work are summarized in Table-1 (for notation please refer to Figure-4). For the Ljunction case, we denote the features by $\mathbf{X}_{q}^{l}$, and for the Tjunction case we use $\mathbf{X}_{q}^{t}$. The first feature of $\mathbf{X}_{q}^{l}$ is $l_{i}$ denoting the distance between the two endpoints. The second feature $\tilde{l}_{i}$ is the sum of two distances between each endpoint and the estimated center of the junction. The angular completion features are adopted from [59]. These feature measures the turning angle of the junction, and the difference of two angles at each end of the completion edgelet. According to [59] the linear combination of these two features is a scale invariant approximation of the elastica energy. For T-junction edgelets, the first feature $l_{i}$ denotes the edge length, the second feature is the ratio between the length of the T-junction edgelet and the length of the occluded gradient edgelet. The third feature measures the deviation of the junction center from the center of the occluding edgelet. The last feature is the squared difference between $\pi / 2$ and the angle of this T-junction.

\subsection{Contour closure potentials $\psi_{\Gamma}$}

The contour closure principle is also difficult to capture by local potential functions. In this paper we approximate the global closure principle by a sequence of connectedness constraints. At an endpoint of an edgelet we can identify two types of such connectedness constraints, each of them is formulated as a set of linear inequalities given follows:

\begin{tabular}{c|c|l}
\hline & image features & description \\
\hline \hline $\mathbf{X}_{q}^{l}(1)$ & $l_{i}$ & effective distance(smooth) \\
$\mathbf{X}_{q}^{l}(2)$ & $\tilde{l}_{i}$ & effective distance (corner) \\
$\mathbf{X}_{q}^{l}(3)$ & $a_{i}\left(\theta_{i, j}+\theta_{i, k}\right)^{2}$ & angular completion \\
$\mathbf{X}_{q}^{l}(4)$ & $b_{i}\left(\theta_{i, j}-\theta_{i, k}\right)^{2}$ & angular completion \\
\hline $\mathbf{X}_{q}^{t}(1)$ & $l_{i}$ & effective distance \\
$\mathbf{X}_{q}^{t}(2)$ & $l_{i} / l_{j}$ & relative distance \\
$\mathbf{X}_{q}^{t}(3)$ & $\frac{\min \left(l_{k}^{a}, l_{k}^{b}\right)}{l_{k}^{a}+l_{k}^{b}}$ & intersection position \\
$\mathbf{X}_{q}^{t}(4)$ & $\left(\theta_{i, k}-\pi / 2\right)^{2}$ & intersection angle \\
\hline $\mathbf{x}_{i}^{d}(1)$ & $l_{i}$ & length of edgelets \\
$\mathbf{x}_{i}^{d}(2)$ & $\log \left(P b_{i}\right)$ & average log(Pb) response \\
$\mathbf{x}_{i}^{d}(3)$ & $l_{i} \log \left(P b_{i}\right)$ & sum of log(Pb) response \\
\hline
\end{tabular}

TABLE 1

Summary of image features used in junction potential functions (c.f. Fig-4). For completeness, we also include the unary data term features $\mathrm{x}_{i}^{d}$.

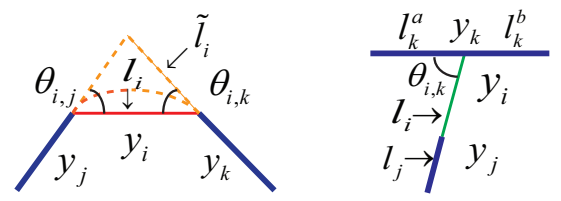

Fig. 4. Two types of completion edgelets. Left: an L-junction edgelet and its image feature description; Right: a T-junction edgelet and its image feature description.

(1) Completion Constraints ensure that no completion edgelet can be active without both of its connected gradient edgelets being active. That is, at either endpoint of a completion edgelet $y_{i}, i \in V_{c}$, its neighboring gradient edgelet $y_{j}, j \in V_{g}$ should satisfy the inequality,

$$
y_{i} \leq y_{j}, \quad \forall i \in V_{c}, j \in V_{g},(i, j) \in C^{P} .
$$

(2) Extension Constraints ensure that if a gradient edgelet is active, then at least one of its connected completion edgelets should be active such that it can be extended. Formally, at either endpoint of a gradient edgelet $y_{j}, j \in V_{g}$, all the edgelets incident to that endpoint, which form a clique $q \in C^{H}$, should satisfy the inequality,

$$
y_{j} \leq \sum_{i \in q \cap V_{c}} y_{i}, \quad \forall j \in V_{g}, q \in C^{H}
$$

Figure-5 illustrates some configurations that either satisfy all the above constraints, or violate one of these constraints. Together, inequalities-(4) and -(5) ensure the connectedness of contours, and hence in the solution space, no contour will be extracted with loose ends. Each of the above inequality constraints can be coded as a polynomial term which equals to 0 when the inequity is satisfied, and equals to $M$ which is a sufficiently large number, otherwise. The inequity-(4) is coded as $\mathrm{M}\left(1-y_{j}\right) y_{i}$, and the inequity (5) is coded as $\mathrm{My}_{j} \prod_{i \in q \cap V_{c}}\left(1-y_{i}\right)$. We collectively represent all the connectedness constraints by the contour closure potentials, 


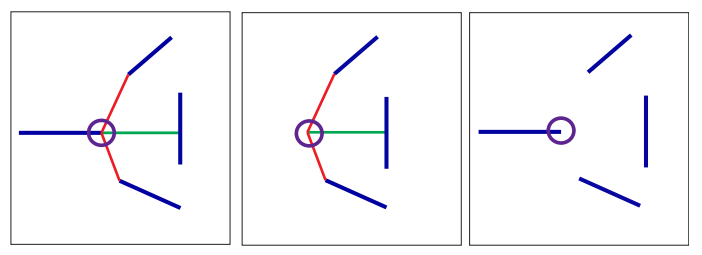

Fig. 5. Examples of valid/invalid configurations w.r.t. the contour closure potential. Blue: Gradient edgelets; Red/Green: Completion edgelets. Left: A valid configuration which satisfies the closure potential. Middle: A configuration which violates the completion constraint (i.e. Eq-(4)). Right: A configuration which violates the extension constraint (i.e. Eq-(5)).

$$
\begin{aligned}
& \sum_{q \in C^{P} \cup C^{H}} \psi_{\Gamma}\left(\mathbf{Y}_{q}\right)= \\
& \mathrm{M}\left(\sum_{\substack{(i, j) \in C^{P} \\
i \in V_{c}, j \in V_{g}}}\left(1-y_{j}\right) y_{i}+\sum_{\substack{q \in C^{H} \\
j \in V_{g}}} y_{j} \prod_{i \in q \cap V_{c}}\left(1-y_{i}\right)\right),
\end{aligned}
$$

where $\mathrm{M}$ is a very big positive number. Note that $\psi_{\Gamma}\left(\mathbf{Y}_{q}\right)=0$, if and only if the corresponding inequality is satisfied.

\subsection{Model complexity potentials $\psi_{M}$}

In natural images, image contours often have multiple levels of details, depending on the scale at which a scene is perceived. To reflect this, we introduce a fourth-type potential function, which can be viewed as adding a preference towards reducing the total effective length of completion edgelets, hence controls the overall model complexity:

$$
\psi_{M}\left(y_{i}, \mathbf{x}_{i}^{m}\right)=\tau \mathbf{w}_{m}^{T} \mathbf{x}_{i}^{m} y_{i},
$$

where $\mathbf{x}_{i}^{m}$ is a feature representing the effective length of the completion edgelet $y_{i}, \mathbf{w}_{m}$ the (negative valued) weighting coefficients, and $\tau$ is a user-specified global scalar controlling the overall model complexity. Details will be given in the following sections. Note that this term is simply a weighted "label cost" used in [8], which is a global higher-order term.

\subsection{Energy function simplification}

The high order terms in (3) make the energy minimization problem difficult to solve. Fortunately, due to the speciality of our potential functions, especially noting that the $y_{i} \mathrm{~s}$ are boolean variables, we realize that when Eq-(4) is true, then the cubic-term triplet junction potential in Eq. (3) can be reduced to linear terms, i.e.

$$
\mathbf{w}_{J}^{T} \mathbf{X}_{q} y_{i} y_{j} y_{k}=\mathbf{w}_{J}^{T} \mathbf{X}_{q} y_{i}, \quad \forall i \in\left(q \cap V_{c}\right), q \in C^{J} .
$$

This reduction is significant, as it greatly simplifies our energy function. Now, except for the higher-order terms of $\psi_{\Gamma}$, all the other terms are reduced to be unary and linear. Moreover, even the higher-order terms $\psi_{\Gamma}$ are in linear form, because they are nothing but the linear inequalities in Eq-(4) and Eq-(5).

In summary, given input $\mathbf{X}$, the problem of maximizing the conditional probability (1) can be transformed into an Integer

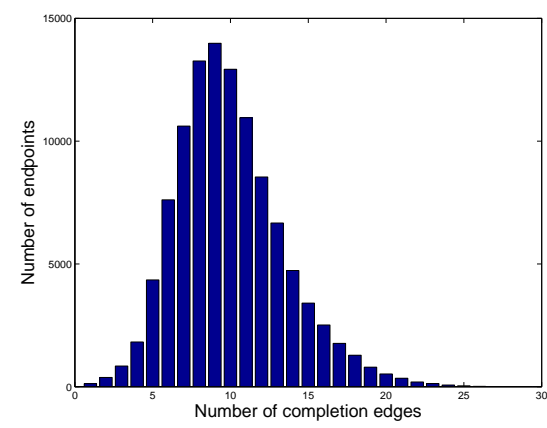

Fig. 6. The histogram of the number of completion edgelets connected to one endpoint on the BSDS dataset. We can see that there are some junctions with dense connections.

Linear Program (ILP):

$$
\min _{\mathbf{Y}} \alpha \sum_{i=1}^{N} \mathbf{w}_{d}^{T} \mathbf{x}_{i}^{d} y_{i}+\sum_{q \in C^{J} \wedge i \in q \cap V_{c}} \mathbf{w}_{s}^{T} \mathbf{X}_{q} y_{i}-\tau \sum_{i \in V_{c}} \mathbf{x}_{i}^{m} y_{i}
$$$$
\text { s.t. } \quad \psi_{\Gamma}\left(\mathbf{Y}_{q}\right)=0, \quad \forall q \in C^{P} \cup C^{H} \text {. }
$$

The constraints are simply the linear inequalities in Equation (4) and (5).

\section{INFERENCE}

After combining all potential functions together, we obtain a (very) higher-order CRF model. To directly solve this higherorder CRF is very difficult. First of all, unlike previous CRF models, our graph construction allows much larger cliques to form. Therefore, it is non-trivial to apply message-passing algorithms, such as Loopy BP, to the problem. Secondly, it is shown in Section 5.1 that the higher-order inequality constraints in $\psi_{\Gamma}$ make the whole energy function non-submodular under very mind conditions. This non-submodularity and highorder properties of our energy function prove difficult to apply graphcut [4] or QPBO [17] for inference.

Therefore, a customized inference algorithm based on linear relaxation is proposed in Section 5.2. The feasibility and efficiency of this algorithm is discussed in Section 5.3 and Section 6.3.

\subsection{Properties of the proposed energy function}

First, unlike previous boundary CRF models, our graph construction allows large-sized cliques to form. As shown in Figure 6, the mean value of vertex degrees is around 10 and the maximum is 25 for 100 BSDS images. Secondly, although most part of the energy function is linear, the higherorder constraints in $\psi_{\Gamma}$ make the whole energy function nonsubmodular. It can be shown that if there exist two singleconnected contours which share some edgelets, then the energy function is non-submodular. See the appendix A for detailed proof.

Proposition 1. If there are at least two single-connected contours intersecting with each other, then the energy function in Eq.(1) is non-submodular. 
Empirically we also observe that the assumption is satisfied in most cases. In the following subsection, we propose a novel optimization method based on the cutting-plane and coordinate descent methods.

\subsection{Algorithm description}

For clarity of presentation, the energy minimization problem is formulated as a standard integer program:

$$
\begin{array}{cl}
\min _{Y} & c^{T} \mathbf{Y} \\
\text { s.t. } & A \mathbf{Y} \leq b \\
& y_{i} \in\{0,1\}, \forall i \in V,
\end{array}
$$

where $\mathbf{Y}=\left\{y_{i}, i \in V\right\}$ is the labels of all edgelets. The parameter vector $c$ is the weights of edgelets, and $A, b$ parameterize linear constraints for the connectedness constraints.

While there exist many general-purpose off-the-shelf ILP solvers, such as those based on branch-and-bound, they are incapable to handle large-scale problems such as our case (where there typically are thousands of variables and constraints to be solved). We therefore propose a tailored optimization approach, which combines the ideas of cutting-plane and coordinate-descent.

Our inference algorithm is shown as Algorithm 1. We firstly solve a linear relaxation of the original integer program. Usually, after each round some variables have fractional values and we sequentially add more constraints such that those fractional variables have to be boolean. Unlike the conventional cutting-plane method, we directly add the integral constraints to a small number of fractional variables instead of searching for a cut. In particular, we randomly select $N_{\max }$ fractional variables, and add their integer constraints into Eq. (8). Then we solve a mixed integer linear programming (MILP) that generates integer solutions to the selected fractional variables. Once a solution to the MILP is found, we fix the values of the selected subset of variables and search an optimal configuration for the remaining variables iteratively.

Since this algorithm is not guaranteed to find global optimum, it is necessary to assess the quality of solutions. Section 5.3 first shows that the algorithm can always find a feasible solution to the integer program. Then Section 6.3 shows that the solutions are also close to the optimal, and can be computed efficiently for our problem.

\subsection{Feasibility of Algorithm 1}

A solution of our algorithm always satisfies connectedness constraints since all the constraints are present in the inference process. In other words, the solution will correspond to topologically correct contours. However, it is not self-evident why the algorithm can always produce a feasible solution. Note that some of the labels are greedily determined when others still have fractional values. Therefore it seems that there could be an infeasible MILP such that Algorithm 1 cannot find a solution at all. The following proposition excludes this possibility:

Proposition 2. Algorithm 1 can always find a feasible solution of $E q(9)-(11)$.

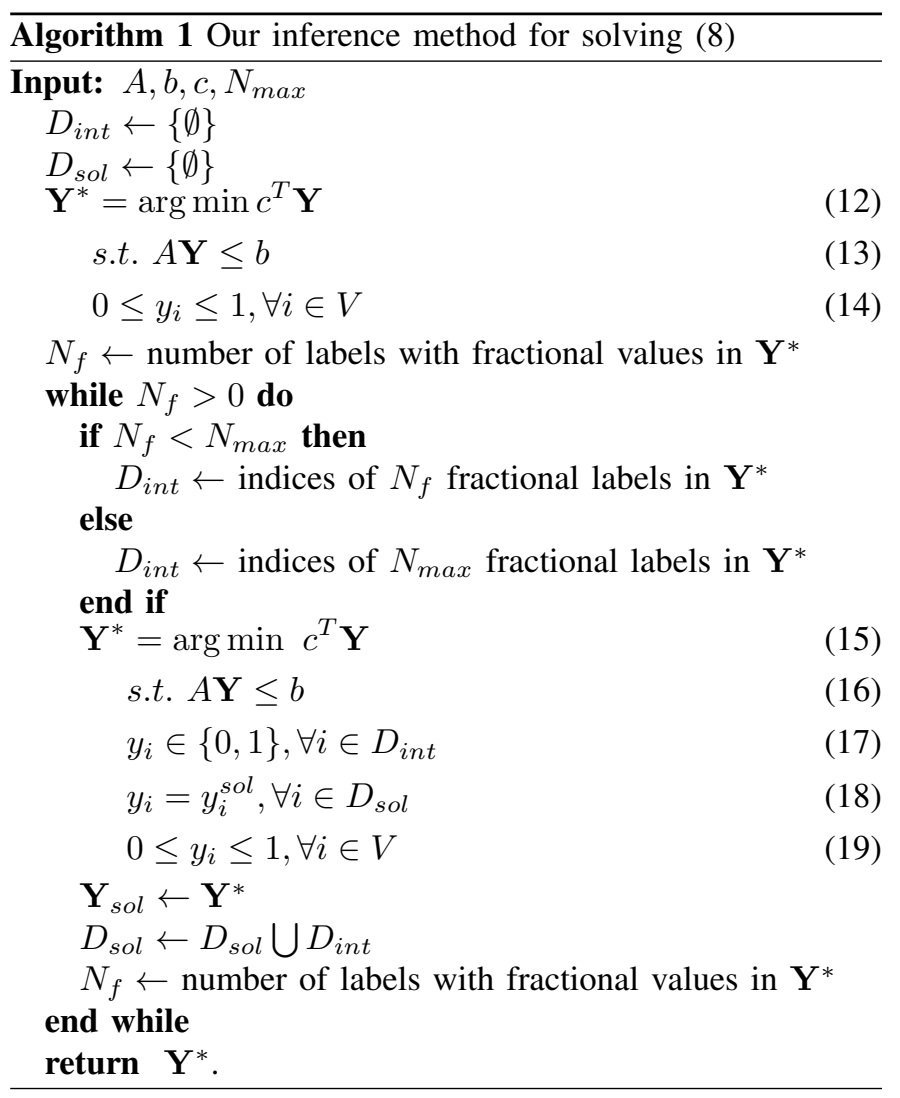

Proof: First, if the LPR and each MILP the algorithm needs to solve is feasible, our inference can find a solution in at most $\left\lceil\frac{N}{N_{\max }}\right\rceil$ iterations. where $\lceil x\rceil$ is the ceil function which return the minimum integer number larger or equal to $x . N$ is the number of variables. $N_{\max }$ denotes the maximal number of integer dimensions that the subroutine can efficiently solve at one time. The solution, once obtained, satisfies the constraints (10)(11), thus is a feasible solution of the ILP. However, it is not obvious that the the LPR and MILP are feasible. The LPR is feasible because of the trivial solution $\mathbf{Y}^{*}=0$. The feasibility of all MILP can be established by induction. Suppose $Y^{*}$ is the solution of the previous MILP (or the LPR for the first iteration), We will show that the next MILP has at least one solution $\overline{\mathbf{Y}}^{*}=\left\lceil\mathbf{Y}^{*}\right\rceil$. In other words, $\overline{\mathbf{Y}}^{*}$ is obtained by any fractional label in $Y^{*}$ to 1 .

The feasibility of $\overline{\mathbf{Y}}^{*}$ can be established by checking the constraints of MILP. First of all, $\overline{\mathbf{Y}}^{*}$ is binary, therefore the $\mathrm{Eq}(17)$ is satisfied. Second, obviously $\bar{y}_{i}^{*}=y_{i}^{*}$ if $y_{i}^{*} \in\{0,1\}$. Therefore, the Eq (18) is satisfied. Finally, we show that $\overline{\mathbf{Y}}^{*}$ satisfies the inequalities. Since $\mathbf{Y}^{*}$ is the previous solution, these inequities are all satisfied:

$$
\begin{aligned}
& y_{i}^{*} \leq y_{j}^{*}, \quad \forall i \in V_{c}, j \in V_{g},(i, j) \in C^{P} . \\
& y_{j}^{*} \leq \sum_{i \in q \cap V_{c}} y_{i}^{*}, \quad \forall j \in V_{g}, q \in C^{\Gamma} \\
& 0 \leq y_{i}^{*} \leq 1, \quad \forall i \in V
\end{aligned}
$$

$\mathrm{Eq}(23)(25)$ are the connectedness constraints dissembled from 


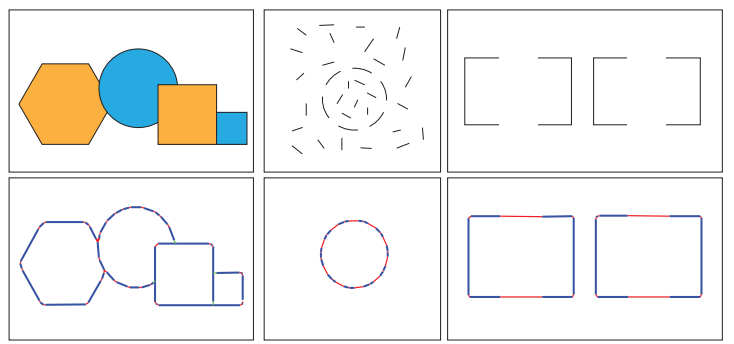

Fig. 7. Top row: three synthetic test images (from left to right: occlusion, clutter, closure). Bottom row: Our results. The lines in blue indicate the active gradient-edges; The lines in red indicate the active L-junction edgelets, and the lines in green indicate the active T-junction edgelets.

$\mathrm{Eq}(19)$. It is straightforward to verify that

$$
\begin{aligned}
& \left\lceil y_{i}^{*}\right\rceil \leq\left\lceil y_{j}^{*}\right\rceil, \quad \forall i \in V_{c}, j \in V_{g},(i, j) \in C^{P} \\
& \left\lceil y_{j}^{*}\right\rceil \leq \sum_{i \in q \cap V_{c}}\left\lceil y_{i}^{*}\right\rceil, \quad \forall j \in V_{g}, q \in C^{\Gamma} \\
& 0 \leq\left\lceil y_{i}^{*}\right\rceil \leq 1, \quad \forall i \in V
\end{aligned}
$$

Therefore, $\overline{\mathbf{Y}}^{*}$ satisfies the $\operatorname{Eq}(16)(19)$ too. Thus, we have proved that MILP will always be feasible. Therefore, Algorithm 1 can always produce a solution.

\section{EXPERIMENTS}

Learning model parameters. The parameters used in the our model, i.e. $\mathbf{w}_{d}, \mathbf{w}_{J}, \mathbf{w}_{m}$ (cf. Eq-(8)), are learned by the logistic regression with a piece-wise learning strategy [62]. For each type of edges, we labeled hundreds of negative and positive samples. The logistic regression is then used to determine the optimal parameters. Cross-validation is used to choose the global parameter $\alpha$ in unary terms (Eq-(8)).

\subsection{Tests on synthetic images}

In order to validate our contour completion model, we test it on a number of purposely designed synthetic images. These images are commonly used in cognitive vision.

Figure-7 shows some examples where our method performs nearly perfectly, extracting multiple closed contours regardless of occlusion (1st image), clutter (2nd image), and prefers closed contours (3rd image), just as we expect. In this figure, we give the raw outputs of our method, where different colors are used to indicate different types of edges: blue stands for gradient edgelets (G-edge); red stands for L-junction edgelets (L-edge); green stands for T-junction edgelets (T-edge). Note that the third image is often used for demonstrating Kanizsa's visual illusionary contours. Our model shows an evident preference of closed contours, similar to human's perception. The L-junction edgelets in-between are turned "on" because they lead to a lower cost to the energy function.

\subsection{Tests on natural images}

Example results. We test our model on BSDS300, and also some other natural images (such as the Weizmann horse dataset [3] etc). Satisfactory results are obtained. Figure-9 gives some sample results of our method, with both raw outputs and the final contour maps displayed. More contour maps overlaid on BSDS300 images can be found in Figure-8. It seems that our method produces very clean, and also connected contours. Even though each of these contours does not necessarily correspond to a semantically meaningful surface region (which would require a higher level of vision processing such as figure-background segmentation), our results do improve over $\mathrm{Pb}$ results significantly, and will be helpful for later-stage high-level vision processing. Our Matlab implementation running on a $2.1 \mathrm{GHz}$ Intel Core Duo CPU, takes about 5 minutes to process a BSDS image (excluding the time used for $\mathrm{Pb}$ detection).

Model complexity. In this experiment, we aim to test the effect of tuning our model-complexity parameter $\tau$. By tuning this parameter, we obtain a series of results, each with a different level of details (complexity). Some examples are shown in Figure-10. Clearly, when $\tau$ is larger, the extracted contour images will have less details, and vice versa. It is worth noting that, in both cases, the connectedness of contours is always maintained, thanks to our hard closure constraints used in the inference. This is in sharp contrast to local methods such as $\mathrm{Pb}$, for which increasing their threshold tends to yield more-fragmented contours.

The closure potentials. This paper's key insight is about the closure principle. In this experiment, we deliberately exclude the two sets of inequality constraints and run inference again. Without the inequities, the probability of each edge is determined solely by its weight.

We have tested two cases, one with the closure potentials, and one without, on all the 300 images in BSDS300. Figure-11 gives a statistical comparison using the precision-recall curves. The effects of the closure potentials are evident.

\subsection{Effectiveness of the inference algorithm}

We demonstrate the effectiveness of the inference algorithm on 100 BSDS images. The quality of a solution is estimated by computing the ratio of solution energy over the energy lower bound obtained by linear programming relaxation. Since in our model energies have negative values, the ratio is a number between zero and one. The histogram of energy ratios is shown in the left half of Figure 12. It shows that the energy of our solutions are very close to the lower bounds. The average ratio is 0.982. Second, we show that the number of MILP iterations is far less than the theoretical upper bound $\left[\frac{N}{N_{\max }}\right]$. As shown in the right half of Figure 12, our inference algorithm only needs to solve up to 6 MILP to obtain a solution while the upper bound is around 100 iterations. This is not surprising as variables are coupled by the linear constraints. When one set of variables are fixed to integers, many other variables will have integer value due to these constraints.

We also tried the multi-start procedure to further minimize the solution energy. The procedure selects ten different sets of the variables for branch in Algorithm 1. Therefore, ten solutions will be obtained by the procedure. The solution with the minimal energy is selected as the final solution. The 


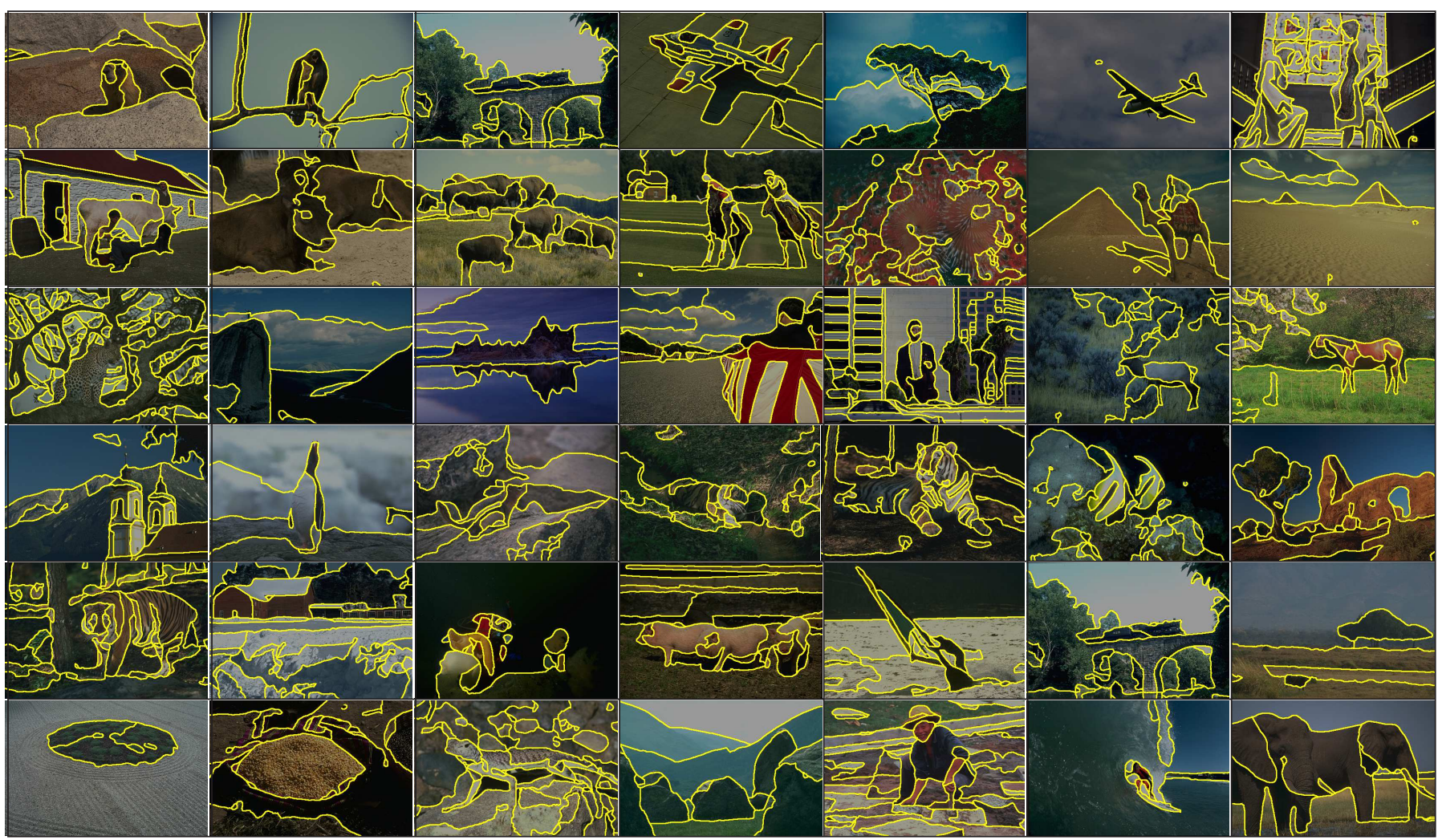

Fig. 8. Contours overlaid with the input images from the BSDS300 dataset. Best viewed in color.

average energy ratio increased to 0.994 . Although the energy is a little more close to global optimal, the contour outputs are little different from those obtained by Algorithm 1. Therefore, we think the Algorithm 1 is enough to obtain a good solution, and the multi-start procedure is not employed for the rest of the experiments.

\subsection{Benchmark with existing methods}

We compared our method with other existing methods for contour extraction and completion. Some example results are shown in Figure-13 for visual evaluation. Compared with $\mathrm{Pb}$, Ren et al.'s CRF and the contour-cut algorithm [24], our method seems to produce better results in terms of contour connectedness. Of course, such a comparison may be seen unfair, as it is not other algorithms' intention to generate connected contours. Nevertheless, we find our results visually more pleasing, which perhaps suggest that our results are closer to human perception. We also did an overall statistical comparison among these algorithms, based on BSDS300 benchmark. The precision-recall curves are plotted in Figure14. Our new model outperforms Ren et al.'s CRF model by a clear margin. In the high-precision regime, our model achieves comparable result with the contour-cut algorithm (using its top 10 contours only), but in the high-recall regime (this regime is most useful for object recognition [25]) our method's performance is more consistent. We did not include Kokkinos' method [25] in the comparison, because he used a dedicated local edge detector (instead of $\mathrm{Pb}$ ).
Our method is further tested on the BSDS500 dataset which has additional 200 test images. As shown in Figure 15 the general trends of the results are similar to those in BSDS300. Our model achieves the same F-value 0.67 as the $\mathrm{Pb}$ detection. However, our model is advantageous when the recall rate is neither too high nor too low. This is understandable since the priors of our model, i.e. smoothness, connectedness are most effective in the mid-recall range. When the recall rate is high, the contours obtained from thresholding $\mathrm{Pb}$ detection have very small gaps. Therefore, even when our model connects the contours correctly, the recall rate will not benefit much. The incorrect completions however, will lower the precision. In the low recall, high precision region, salient gradient edges are sparse, and it becomes more difficult for our model to connect these sparse edges. According to [24], contours associated with first 20 eigenvalues are selected as outputs. Their performance is enhanced by spectral information in the high precision region.

Contour Rand index. Human are sensitive to the completion of contours. For example, if the central point of a line segment is removed, human would recognize that as a broken line. In contrast, it is less noticeable if a point is removed from either end of the line segment. The precisionrecall metric, however, are not affected in either case. As a complementary measure to PR curves, we adapt the wellknown Rand Index metric [51] to the contour case. Rand Index is widely used for measuring clustering error. In this paper, all connected contour points are considered in the same cluster/group, thus the change of connectedness condition 


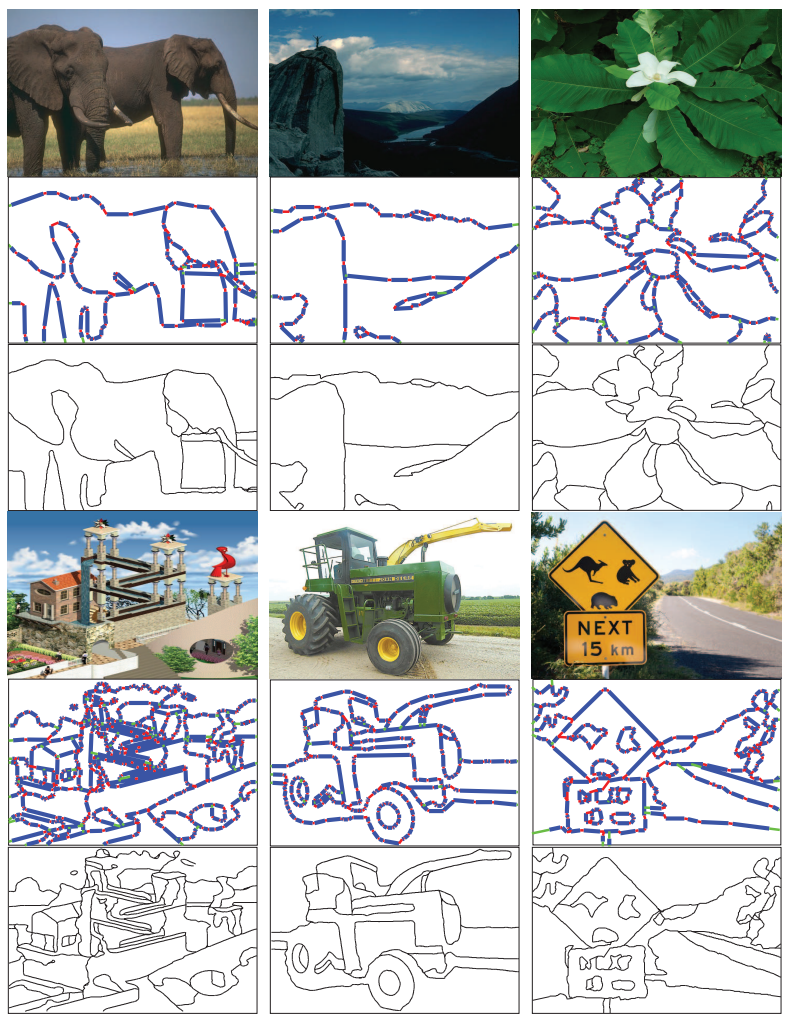

Fig. 9. Sample results of our method on natural scene images. For every three rows, top row: the input images; middle row: our method's raw outputs (blue: G-edge; red: L-edge; green: Tedge); bottom row: Our method's final contour maps. (Better viewed on screen with zoom-in).

will lead to different clustering. We call our new metric the Contour Rand Index (CRI). This metric should be used in conjunction with precision and recall metrics for a more complete assessment of contour quality.

The Rand index is defined as follows: let $S=\left\{e_{1}, e_{2} \ldots e_{n}\right\}$ be a set of elements, $C=\left\{C_{1}, C_{2} \ldots C_{r}\right\}$ and $\tilde{C}=$ $\left\{\tilde{C}_{1}, \tilde{C}_{2} \ldots \tilde{C}_{t}\right\}$ are two partitions of $S$. For example, $C_{i}$ s are non-intersecting subsets of $S$. Let $a$ be the number of pairs of elements in $S$ which are partitioned into the same subset in both $C$, and $\tilde{C} ; b$ be the number of pair of elements which are partitioned into different subsets both in $C$, and in $\tilde{C}$. The number of all pairs of elements is $p$. Rand index is,

$$
R I=\frac{a+b}{p}
$$

To adapt the Rand index to the contour grouping problem, we make use of the groundtruth contours in the BSDS dataset. The objective is to compute Rand index as the similarity of grouping between a contour image and human labeling. However, two problems need to be solved. First we need to determine the elements for grouping. Rand index is defined on the same set of elements. Apparently, a groundtruth image and a contour image have different sets of contour points. Second, we need to determine which elements are in the same group, given a contour or groundtruth image.

To address the first problem, bipartite matching is carried out between the groundtruth and contour image. Every

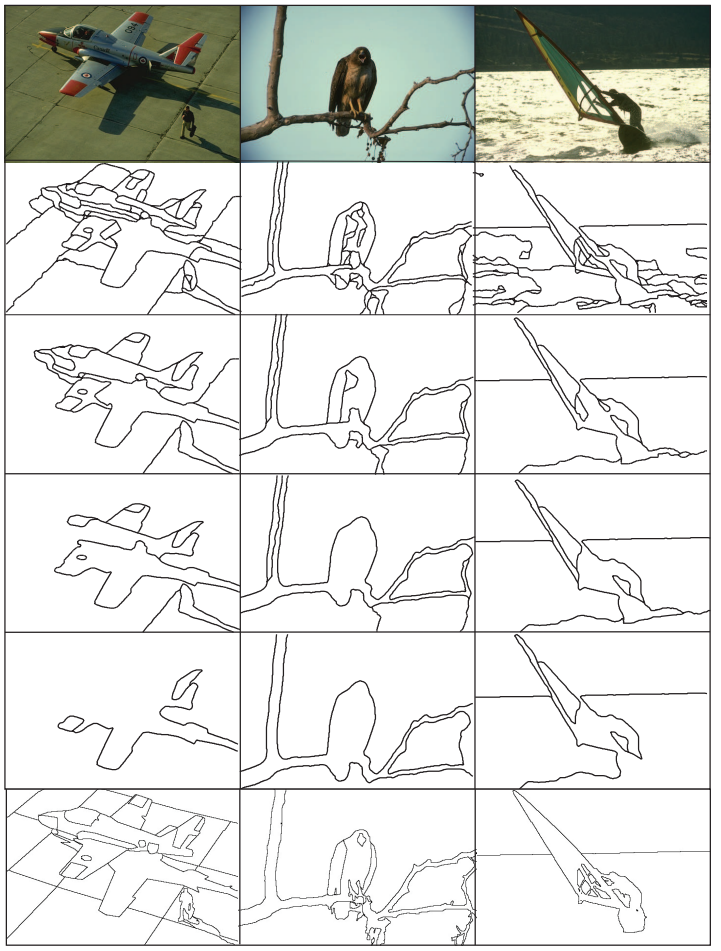

Fig. 10. Effect of tuning the model complexity parameter $\tau$. Row 1: The input images. Row 2 5: Our method's outputs when $\tau=0,1,2,3$. As $\tau$ increases, the extracted contour images contain less details, yet connectedness is well maintained. Row 6: Edge maps labeled by human.

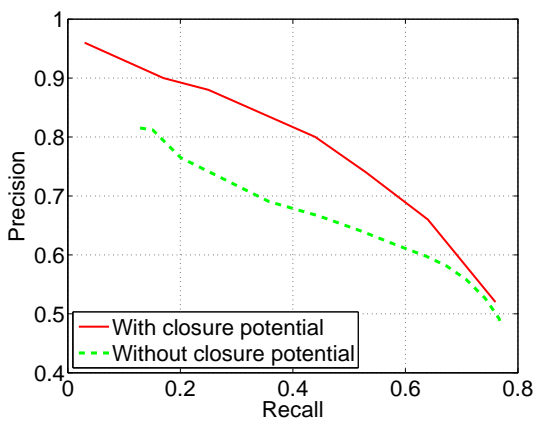

Fig. 11. Effect from the closure potentials. It is clear that the closure-potentials substantially boost model's overall performance.
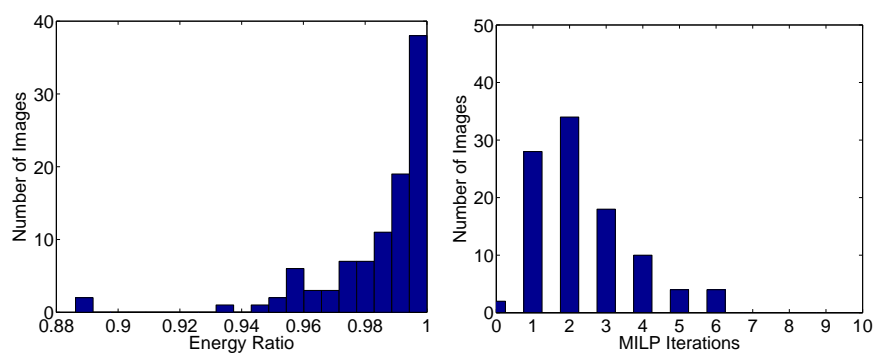

Fig. 12. The left figure shows the histogram of the ratios of solution energy over lower bound energy. The right figure shows the histogram of MILP iterations per image. The experiment is conducted on 100 BSDS images. 


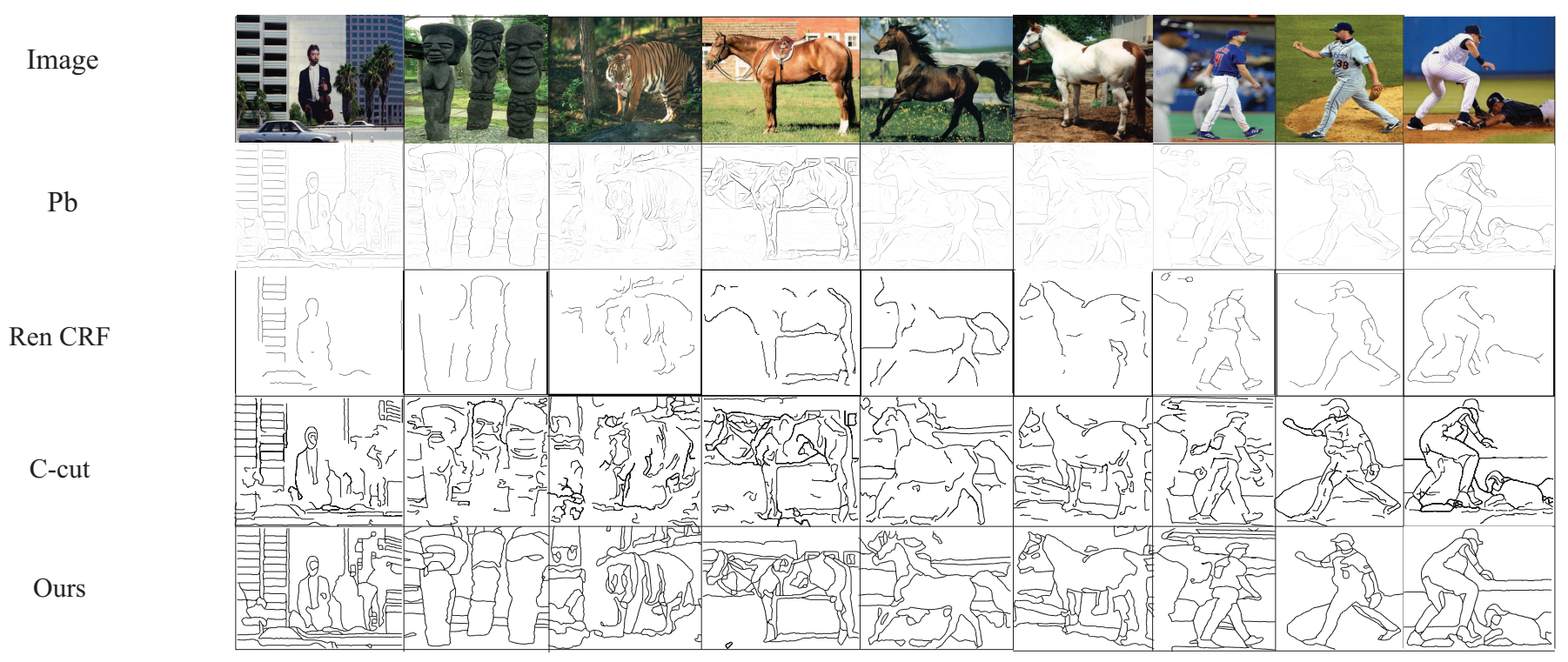

Fig. 13. Methods comparisons on natural scene images: Top row: sample images from the BSDS dataset [2], the Weizmann horse dataset [3], and baseball player dataset[43]. Other rows (from top to bottom): the Pb detector, Ren's CRF (reproduced from [55]), the contour-cut method, and our method $(\tau=0.5)$.

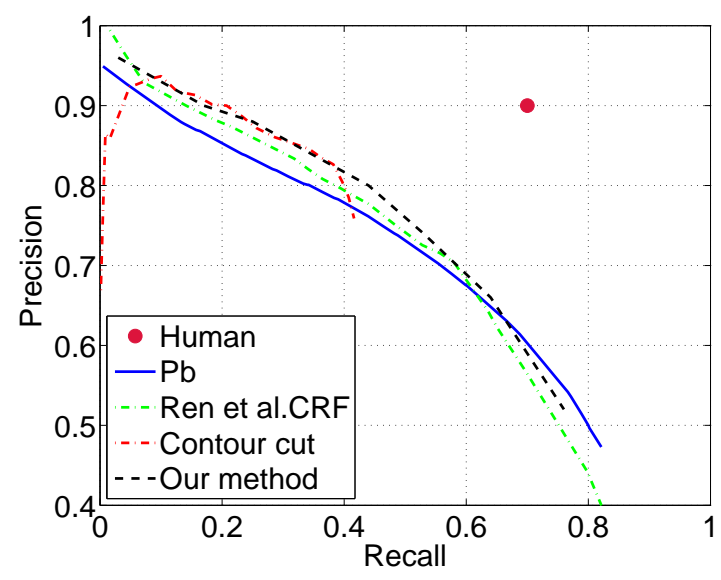

Fig. 14. Precision-Recall curves for 4 methods on the BSDS300 dataset (Better viewed on screen).

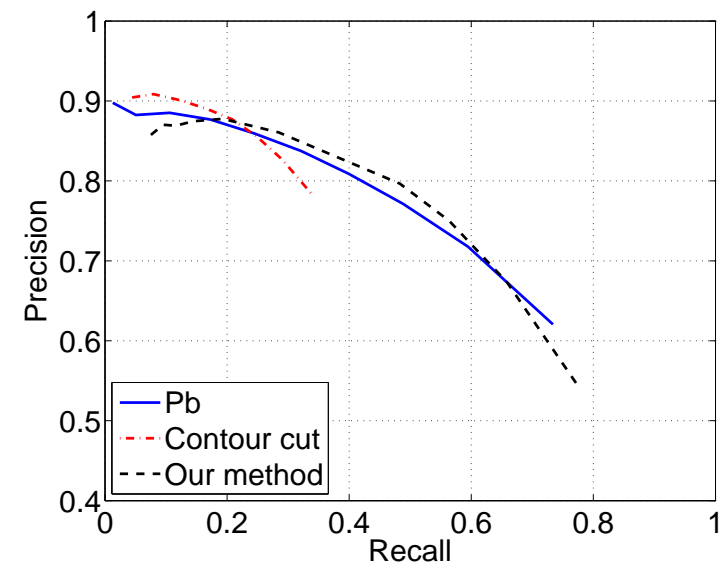

Fig. 15. Precision-Recall curves on the BSDS500 dataset (Better viewed on screen). matched pair of points is considered as one element in Rand index. To address the second problem, we group contours based on the connectivity. We search for 8-connected contour points in a contour image as a group, and assign these connected points a common group number. Searching stops when all the contour points have a group number. The same process is carried out for groundtruth. A pair of points with same group number in the test image is correctly grouped if their matched points in the groundtruth have the same grouping number. If these two points are assigned with different group numbers in the test image, their counterparts in the groundtruth need to have different group numbers. To improve the stability of the metric, we remove the contour groups containing less than 10 image pixels. Note that although all the contour points have a group number, only matched points are taken into account by the metric. Last, the Rand index is averaged across the images and human subjects. In sum, CRI is defined as:

$$
C R I=\frac{\sum_{i} \sum_{j}\left(a_{i, j}+b_{i, j}\right)}{\sum_{i} \sum_{j} p_{i, j}},
$$

where $i$ is the index of human subjects and $j$ is the index of test images.

To demonstrate the effectiveness of connectedness prior, our method is mainly compared with methods which are also based on the same bottom-up edge detector $(\mathrm{Pb})$. The CRI values are shown in Figure 16. It shows that our model consistently outperforms $\mathrm{Pb}$ in all recall region. Our model's outputs also outperform the contour-cut algorithm. Two recent methods [71], [9] have higher accuracy than $\mathrm{Pb}$. However, without enforcing contour connectedness, they also have lower CRI values. Under optimal threshold, [71] has a CRI of 0.34, and [9] has a CRI of 0.37 . 


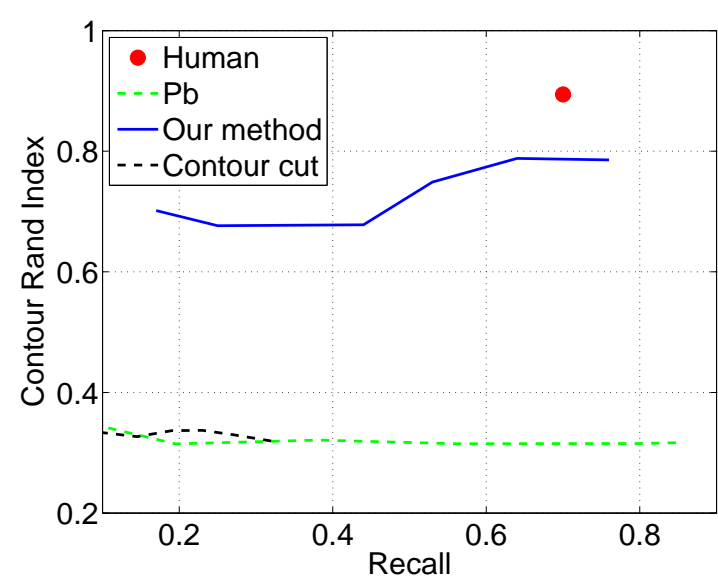

Fig. 16. Performance comparison by Contour Rand Index. Human $\mathrm{CRI}$ is shown as a red dot.

\section{Closing remarks}

Understanding the mechanism for contour-completion holds the promise to develop better-performing image understanding systems. This is however, a very challenging task which is thought to be achieved by segmentation. In this paper, we have presented a purely contour-based CRF method attempting to enforce the contour closure principle, and derived an efficient optimization method to perform approximate inference on the higher-order CRF. The results on natural scene image datasets show that our method can produce highly-completed contours without sacrificing point-wise detection accuracy. We hope this work will provide useful ideas for perceptual grouping research.

\section{ACKNOWLEDGMENTS}

This work is supported by Australia Research Council via an ARC-DP grant to the second author. NICTA is a research center of excellence funded by the Australian Government, in part through ARC. We would like to thank Dr Xiaofeng Ren for providing the code of his CRF model.

\section{REFERENCES}

[1] B. Andres, J. H. Kappes, T. Beier, U. Köthe, and F. Hamprecht Probabilistic image segmentation with closedness constraints. In Proc. International Conference on Computer Vision, 2011.

[2] P. Arbelaez, M. Maire, C. Fowlkes, and J. Malik. Contour detection and hierarchical image segmentation. IEEE Trans. Pattern Analysis and Machine Intelligence, 33(5):898-916, 2011.

[3] E. Borenstein and S. Ullman. Class-specific, top-down segmentation. In Proc. European Conference on Computer Vision, pages 109-124, 2002.

[4] Y. Boykov, O. Veksler, and R. Zabih. Fast approximate energy minimization via graph cuts. IEEE Trans. Pattern Analysis and Machine Intelligence, 23:1222 -1239, 2001.

[5] X. Bresson, P. Vandergheynst, and J.-P. Thiran. A variational model for object segmentation using boundary information and shape prior driven by the mumford-shah functional. International Journal of Computer Vision, 68(2):145-162, 2006.

[6] J. Canny. A computational approach to edge-detection. IEEE Trans. Pattern Analysis and Machine Intelligence, 8(6):679-698, 1986.

[7] D. Cremers, T. Kohlberger, and C. Schnörr. Shape statistics in kernel space for variational image segmentation. Pattern Recognition, 36(9):1929-1943, 2003.

[8] A. Delong, A. Osokin, H. N. Isack, and Y. Boykov. Fast approximate energy minimization with label costs. International Journal of Computer Vision, 96(1):1-27, 2012.
[9] P. Dollár and C. Zitnick. Structured forests for fast edge detection. In Proceedings of the IEEE International Conference on Computer Vision, pages 1841-1848, 2013.

[10] J. H. Elder and S. W. Zucker. Computing contour closure. In Proc. European Conference on Computer Vision, pages 399-412, 1996.

[11] A. C. Gallagher, D. Batra, and D. Parikh. Inference for order reduction in markov random fields. In Proceedings of the 2011 IEEE Conference on Computer Vision and Pattern Recognition, CVPR '11, Washington, DC, USA, 2011. IEEE Computer Society.

[12] D. Geman, S. Geman, C. Graffigne, and P. Dong. Boundary detection by constrained optimization. IEEE Trans. Pattern Analyis and Machine Intelligence, 12(7):609-628, 1990.

[13] S. Geman and D. Geman. Stochastic relaxation, gibbs distributions, and the bayesian restoration of images. Pattern Analysis and Machine Intelligence, IEEE Transactions on, PAMI-6(6):721 -741, nov. 1984.

[14] S. Gould. Multiclass pixel labeling with non-local matching constraints. In $C V P R, 2012$.

[15] C.-e. Guo, S.-C. Zhu, and Y. N. Wu. Primal sketch: Integrating structure and texture. Comput. Vis. Image Underst., 106(1):5-19, 2007.

[16] G. Guy and G. Medioni. Inferring global perceptual contours from local features. In Proc. IEEE Conference on Computer Vision and Pattern Recognition, pages 786-787, 1993.

[17] P. Hammer, P. Hansen, and B. Simeone. Roof duality, complementation and persistency in quadratic 0-1 optimization. Mathematical Programming, 28:121-155, 1984.

[18] B. Hariharan, P. Arbelaez, L. Bourdev, S. Maji, and J. Malik. Semantic contours from inverse detectors. In Computer Vision (ICCV), 2011 IEEE International Conference on, pages 991 -998, 2011

[19] B. K. P. Horn. The curve of least energy. ACM Trans. Math. Softw. 9(4):441-460, Dec. 1983.

[20] H. Ishikawa. Higher-order clique reduction in binary graph cut. In Computer Vision and Pattern Recognition, 2009. CVPR 2009. IEEE Conference on, pages $2993-3000$, june 2009.

[21] P. Isola, D. Zoran, D. Krishnan, and E. H. Adelson. Crisp boundary detection using pointwise mutual information. In Computer VisionECCV 2014, pages 799-814. Springer, 2014.

[22] A. K. Jain. Fundamentals of digital image processing. Prentice-Hall, Inc., Upper Saddle River, NJ, USA, 1989.

[23] M. Kass, A. Witkin, and D. Terzopoulos. Snakes - active contour models. International Journel of Computer Vision, 1(4):321-331, 1987.

[24] R. Kennedy, J. Gallier, and J. B. Shi. Contour cut: identifying salient contours in images by solving a hermitian eigenvalue problem. In Proc. IEEE Conference on Computer Vision and Pattern Recognition, pages 2520-2527, 2011.

[25] I. Kokkinos. Highly accurate boundary detection and grouping. In Proc. IEEE Conference on Computer Vision and Pattern Recognition, pages 2520-2527, 2010.

[26] I. Kovacs and B. Julesz. A closed curve is much more than an incomplete one - effect of closure in figure ground segmentation. PNAS,USA, 90(16):7495-7497.

[27] P. Kovesi. Matlab and octave functions for computer vision and image processing. http://www.csse.uwa.edu.au/ pk/research/matlabfns.

[28] P. Kovesi and P. Kovesi. Image features from phase congruency. Videre: Journal of Computer Vision Research, MIT Press, 1(3).

[29] P. Krähenbühl and V. Koltun. Efficient inference in fully connected crfs with gaussian edge potentials. arXiv preprint arXiv:1210.5644, 2012.

[30] R. Ladicky, C. Russell, P. Kohli, and P. Torr. Inference methods for crfs with co-occurrence statistics. International Journal of Computer Vision, pages $1-13,2012$

[31] V. S. Lempitsky, P. Kohli, C. Rother, and T. Sharp. Image segmentation with a bounding box prior. In ICCV, pages 277-284, 2009.

[32] T. Leung and J. Malik. Contour continuity in region based image segmentation. In Proc. European Conference on Computer Vision, pages $544-559,1998$

[33] A. Levinshtein, C. Sminchisescu, and S. Dickinson. Optimal contour closure by superpixel grouping. In Proceedings of the 11th European conference on Computer vision, pages 480-493, 2010.

[34] Z. Li. A neural model of contour integration in the primary visual cortex. Neural Computation, 10:903-940, 1998

[35] J. Lim, C. Zitnick, and P. Dollár. Sketch tokens: A learned mid-level representation for contour and object detection. In Proceedings of the IEEE Conference on Computer Vision and Pattern Recognition, pages $3158-3165,2013$.

[36] T. Lindeberg. Scale-space theory: A basic tool for analysing structures at different scales. Journal of Applied Statistics, 21(2):224-270, 1994.

[37] S. Mahamud, L. R. Williams, K. K. Thornber, and K. L. Xu. Segmentation of multiple salient closed contours from real images. IEEE Trans. Pattern Analysis and Machine Intelligence, 25(4):433-444, 2003.

[38] M. Maire, P. Arbelaez, C. Fowlkes, and J. Malik. Using contours to detect and localize junctions in natural images. 2008 IEEE Conference 
on CVPR, Vols 1-12, pages 611-618, 2008.

[39] D. Marr. Vision: A Computational Investigation into the Human Representation and Processing of Visual Information. Henry Holt and Co., Inc., 1982.

[40] D. Marr and E. Hildreth. Theory of edge detection. Proceedings of the Royal Society of London. Series B. Biological Sciences, 207(1167):187217, 1980.

[41] D. R. Martin, C. C. Fowlkes, and J. Malik. Learning to detect natural image boundaries using local brightness, color, and texture cues. IEEE Trans. Pattern Analysis and Machine Intelligence, 26(5):530-549, 2004

[42] Y. Ming, H. Li, and X. He. Connected contours: A new contour completion model that respects the closure effect. In Proc. CVPR, pages $829-836$, june 2012.

[43] G. Mori, X. Ren, A. A. Efros, and J. Malik. Recovering human body configurations: combining segmentation and recognition. In Proc. IEEE Conference on Computer Vision and Pattern Recognition, pages 326333, 2004.

[44] M. Niss. History of the lenz-ising model 1950-1965: from irrelevance to relevance. Archive for History of Exact Sciences, 63:243-287, 2009.

[45] S. E. Palmer. Vision science : photons to phenomenology. MIT Press, Cambridge, Mass., 1999.

[46] S. E. Palmer, J. L. Brooks, and R. Nelson. When does grouping happen? Acta Psychologica, 114(3):311-330, 2003.

[47] G. Papari and N. Petkov. Edge and line oriented contour detection: State of the art. Image and Vision Computing, 29(2-3):79-103, 2011.

[48] P. Parent and S. W. Zucker. Trace inference, curvature consistency, and curve detection. IEEE Transaction on Pattern Analysis and Machine Intelligence, 11:823-839, 1989.

[49] S. B. Piotr Dollar, Zhuowen Tu. Supervised learning of edges and object boundaries. In Proc. IEEE Conference on Computer Vision and Pattern Recognition, pages 1964-1971, 2006.

[50] V. Prisacariu and I. Reid. Nonlinear shape manifolds as shape priors in level set segmentation and tracking. In Computer Vision and Pattern Recognition, 2011 IEEE Conference on, pages 2185 -2192, 2011.

[51] W. M. Rand. Objective criteria for the evaluation of clustering methods. Journal of the American Statistical Association, 66(336):846-850, 1971.

[52] X. Ren. Multi-scale improves boundary detection in natural images. In Proceedings of the 10th European Conference on Computer Vision, pages 533-545, 2008.

[53] X. Ren and L. Bo. Discriminatively trained sparse code gradients for contour detection, 2012.

[54] X. F. Ren, C. C. Fowlkes, and J. Malik. Figure/ground assignment in natural images. Computer Vision - Eccv 2006, Pt 2, Proceedings, 3952:614-627, 2006.

[55] X. F. Ren, C. C. Fowlkes, and J. Malik. Learning probabilistic models for contour completion in natural images. International Journel of Computer Vision, 77(1-3):47-63, 2008.

[56] S. Roth and M. J. Black. Fields of experts: A framework for learning image priors. In IEEE Conference on Computer Vision and Pattern Recognition, pages 860-867, 2005.

[57] T. Schoenemann, S. Masnou, and D. Cremers. The elastic ratio: Introducing curvature into ratio-based image segmentation. IEEE TIP, pages 2565-2581, 2011.

[58] A. Sha'asua and S. Ullman. Structural saliency: The detection of globally salient structures using a locally connected network. In ICCV, pages 321-327, 1988.

[59] E. Sharon, A. Brandt, and R. Basri. Completion energies and scale. IEEE Trans. Pattern Analysis and Machine Intelligence, 22(10):1117 $1131,2000$.

[60] J. B. Shi and J. Malik. Normalized cuts and image segmentation. IEEE Trans. Pattern Analysis and Machine Intelligence, 22(8):888-905, 2000.

[61] M. suen Lee and G. Medioni. Grouping into regions, curves, and junctions. Computer Vision Image Understanding, 1999.

[62] C. Sutton and A. McCallum. Piecewise training for structured prediction. Machine Learning, 77:165-194, 2009.

[63] W.-S. Tong and C.-K. Tang. Robust estimation of adaptive tensors of curvature by tensor voting. IEEE Trans. Pattern Anal. Mach. Intell., 27(3):434-449, 2005.

[64] S. Vicente, V. Kolmogorov, and C. Rother. Graph cut based image segmentation with connectivity priors. In Computer Vision and Pattern Recognition, 2008, IEEE Conference on, june 2008.

[65] V. Vineet, J. Warrell, and P. H. Torr. Filter-based mean-field inference for random fields with higher-order terms and product label-spaces. International Journal of Computer Vision, 110(3):290-307, 2014.

[66] S. Wang, T. Kubota, J. M. Siskind, and J. Wang. Salient closed boundary extraction with ratio contour. IEEE Trans. Pattern Analysis and Machine Intelligence, 27(4):546-561, 2005.

[67] L. R. Williams and K. K. Thornber. A comparison of measures for detecting natural shapes in cluttered backgrounds. International Journel of Computer Vision, 34(2-3):81-96, 1999.
[68] O. Woodford, P. Torr, I. Reid, and A. Fitzgibbon. Global stereo reconstruction under second order smoothness priors. In Computer Vision and Pattern Recognition, 2008. CVPR 2008. IEEE Conference on, pages $1-8$, june 2008 .

[69] Y. Wu, Z. Si, H. Gong, and S.-C. Zhu. Learning active basis model for object detection and recognition. International Journal of Computer Vision, 90:198-235, 2010.

[70] Y. N. Wu, Z. Si, H. Gong, and S.-C. Zhu. Learning active basis model for object detection and recognition. Int. J. Comput. Vision, 90(2):198$235,2010$.

[71] S. Xie and Z. Tu. Holistically-nested edge detection. In Proceedings of the IEEE International Conference on Computer Vision, pages 1395$1403,2015$.

[72] Q. Zhu, G. Song, and J. Shi. Untangling cycles for contour grouping. In Proc. International Conference on Computer Vision, pages 793-800, 2007.

[73] C. Zitnick and D. Parikh. The role of image understanding in contour detection. In Proc. IEEE Conference on Computer Vision and Pattern Recognition, pages $622-629$, june 2012 .

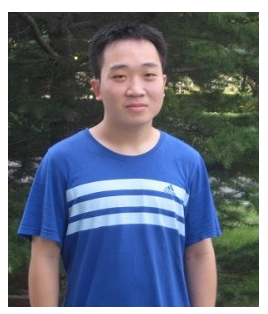

Yansheng Ming obtained his PhD degree from the computer vision and robotics group of Australian National University in 2015. He has been working on several perceptual grouping problems since 2010. He obtained his master' degree from Chinese Academy of Sciences in 2010, and bachelor's degree from Zhejiang University in 2007.

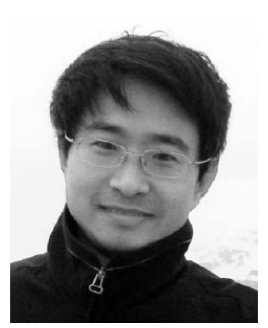

Dr. Hongdong $\mathrm{Li}$ is with the Computer Vision and Robotics Group, CECS, the Australian National University (ANU). His research interests include 3D Computer Vision, Image analysis and pattern recognition, and mathematical optimization. $\mathrm{He}$ is a $\mathrm{Cl}$ and member of ARC Centre of Excellence for Robotic Vision, a former member of the Bionic Vision Australia. Prior to $\mathbf{2 0 1 0}$ he was a Senior Researcher with the National ICT Australia (NICTA), Canberra Labs, and a Fellow with the RSISE, ANU. He is a recipient and co-author of the CVPR Best Paper Award in 2012, ICIP Best Student Paper Award 2014, ICPR Best Student Paper Award 2010, DICTA CSiRA Best Theory Paper Award 2013. He serves on various Programme Committees (Area Chair/Reviewer) for recent ICCV, CVPR, ICIP and ECCV.

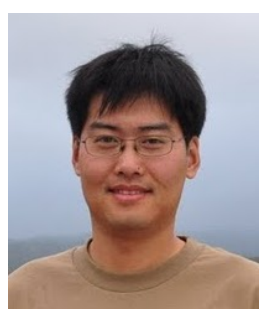

Xuming $\mathrm{He}$ is a Senior Researcher at NICTA Canberra Lab and also an Adjunct Fellow of Engineering Department at the Australian National University (ANU). He received his Ph.D. degree in computer science from the University of Toronto in 2008 . He held a postdoctoral position at the University of California at Los Angeles (UCLA) before joining National ICT Australia (NICTA). His research interests include image segmentation and labeling, visual motion analysis, vision-based navigation, and undirected graphical models. He is a member of IEEE and served as a PC member of IJCAI, CVPR and ICML. 